\title{
Riscos ocupacionais e patologias em cirurgiões-dentistas de centro de especialidades odontológicas
}

\author{
Occupational risks and pathologies in dental surgeons from the Center for \\ Dental Specialties
}

\section{Resumo}

Objetivo: identificar os riscos ocupacionais, danos, agravos e patologias a que estão expostos os cirurgiões-dentistas e verificar a interferência desses eventos na qualidade de vida dos participantes do estudo. Materiais e método: trata-se de um estudo descritivo, com abordagem quanti-qualitativa, realizado no Centro de Especialidades Odontológicas (CEO), localizado na cidade de João Pessoa- PB. Resultados: os resultados revelados por meio do discurso do sujeito coletivo mostraram grandes interferências ocasionadas pelos riscos ocupacionais, em especial a insatisfação com a remuneração e a segurança e higiene no trabalho. Conclusão: apesar de afirmarem que possuem qualidade de vida, a maior parte dos profissionais apresentou sentimentos de insatisfação com a saúde e o trabalho.

Palavras-chave: Riscos ocupacionais. Patologias ocupacionais. Qualidade de vida.

\section{Introdução}

Com a revolução tecnológica ocorrida nos últimos anos, a economia mundial passa por mudanças e adaptações que têm repercutido de forma direta e indireta no modo de produzir. Tais mudanças, pautadas na lógica da competitividade, produtividade e flexibilidade, afetam as condições laborais e de vida dos trabalhadores inseridos nesse contexto.

A precarização do ambiente de trabalho tem como implicação a perda da capacidade laboral do trabalhador e acarreta o aparecimento de doenças ocupacionais ou acidentes de trabalho ${ }^{1}$. Os profissionais de saúde estão imersos em um contexto onde o risco ocupacional faz parte de seu cotidiano. Esses riscos e, consequentemente, o medo do adoecimento e/ou sua concretização interferem diretamente no desempenho dos trabalhadores com repercussões na sua qualidade de vida.

Diante disso, este estudo objetiva identificar os riscos ocupacionais, danos, agravos e patologias aos quais estão expostos os odontólogos do Centro de Especialidades Odontológicas (CEO) no município de João Pessoa-PB e conhecer as interferências na qualidade de vida desses profissionais. 


\section{Materiais e método}

Trata-se de um estudo descritivo com uma abordagem quanti-qualitativa. $\mathrm{O}$ estudo foi realizado no CEO, no ano de 2008. O universo deste estudo foi constituído de 31 cirurgiões-dentistas, e a população foi composta de 24 cirurgiões-dentistas que trabalham no CEO, no centro da cidade de João Pessoa-PB, e concordaram em participar da pesquisa.

Para o desenvolvimento da pesquisa, o pesquisador levou em consideração os aspectos éticos contidos na Resolução 196/96 do Conselho Nacional de Saúde. O protocolo de pesquisa foi aprovado pelo Comitê de Ética em Pesquisa do Centro de Ciências da Saúde da Universidade Federal da Paraíba (UFPB) (Protocolo CEP/CCS № 309/2008).

Para a coleta de dados, foi empregado o recurso da entrevista por ser um meio de interação social e coleta de informações. Os dados obtidos foram trabalhados em duas etapas. Na primeira etapa, foram transcritos e agrupados os dados quantitativos (primeira e segunda partes), que sofreram tratamento estatístico com auxílio do Statistical Package for Social Sciences (SPSS), versão 15.0. Foi feita a análise descritiva das variáveis do estudo, e os resultados foram expressos na forma de tabelas de frequência.

$\mathrm{Na}$ segunda etapa, os dados qualitativos foram transcritos textualmente. Os sujeitos receberam uma codificação de identificação com nomes fictícios, preservando sua identidade, e agrupados com auxílio do QualiQuantiSoft, versão 1.3c.

Nesse contexto, a indicação do Discurso do Sujeito Coletivo fundamenta-se na Teoria das Representações Sociais, por consistir no conjunto de conceitos, afirmações e explicações que são verdadeiras teorias do senso comum, pelas quais as pessoas interpretam a sua realidade e também as realidades sociais, constituindo o pensamento em um verdadeiro ambiente onde se desenvolve a vida cotidiana ${ }^{2}$.

\section{Resultados e discussão}

Fizeram parte do estudo 24 cirurgiões-dentistas, sendo $67 \%$ do sexo feminino e $33,3 \%$ do sexo masculino, com as seguintes características: $70,8 \%$ possuem a graduação como nível máximo de instrução e $29,2 \%$ são especialistas; $45,8 \%$ exercem as suas atividades por 4 horas diárias, $41,7 \%$ as realizam por 6 horas diárias e 12,5\% têm carga de trabalho de 8 horas diárias; $95,8 \%$ dos cirurgiões-dentistas utilizam luvas como equipamentos de proteção individual em suas atividades laborais, $100 \%$ utilizam máscaras, $8,3 \%$ utilizam protetores auriculares, $79,2 \%$ usam gorro, 58,3\% utilizam avental, $66,7 \%$ usam protetor ocular e $4,2 \%$ dos profissionais utilizam propé; todos os profissionais afirmaram estar expostos a riscos biológicos e químicos, entretanto
$50 \%$ mencionaram acometimento em decorrência da exposição biológica e $45,8 \%$ dos riscos químicos; 91,7\% afirmaram exposição a riscos psicossociais, tendo $75 \%$ mencionado acometimento de estresse e outros agravos e $91,7 \%$, exposição a riscos mecânicos, registrando-se que $100 \%$ referiram agravos em decorrência destes; 87,5\% afirmaram exposição a riscos ergonômicos e físicos e $100 \%$ mencionaram acometimento deles decorrente.

Ao analisar a relação de riscos ocupacionais e agravos, danos e doenças ocupacionais (Fig. 1), o coeficiente de correlação de Pearson obtido foi $\mathrm{r}=$ 0,703 . Já o modelo de regressão linear para a relação entre as duas variáveis foi:

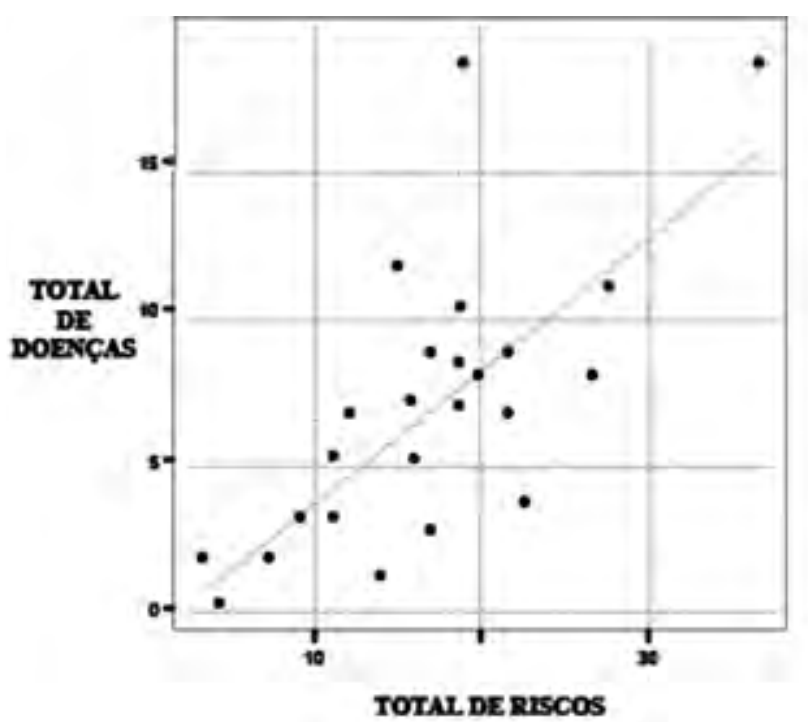

Total de doenças $=0,444$ * Total de riscos $-0,493$ $\left(r^{2}=0,494 ; F=21,458 ; p<0,0005\right)$

Figura 1 - Modelo de regressão linear dos riscos ocupacionais e agravos, danos e doenças ocupacionais referidos pelos cirurgiões-dentistas

Isso indica que, aproximadamente, a cada dois riscos ocupacionais aumenta uma doença de trabalho referida.

$\mathrm{Na}$ avaliação qualitativa, as expressões-chave (ECHs) e as ideias centrais (ICs) que surgiram dos discursos com os cirurgiões-dentistas do CEO contemplam aspectos sobre os danos ocasionados pela exposição aos riscos ocupacionais no seu exercício profissional e a interferência na sua qualidade de vida.

As ICs que foram mencionadas pelos colaboradores consistiram no reconhecimento profissional (Quadro 1). A Odontologia torna-se cada vez mais independente e complexa, devido à expansão das suas práticas de assistência correlacionada com a minimização de custos, que afeta diretamente o comportamento dos cirurgiões-dentistas ${ }^{3}$. 
Quadro 1 - IC 1 - Reconhecimento profissional como fator de interferência da qualidade de vida dos cirurgiões-dentistas

\begin{tabular}{|l|l|}
\hline 1 a IDEIA CENTRAL & \multicolumn{1}{c|}{ Discurso do Sujeito Coletivo } \\
\hline Não sou reconhecido & Na área de saúde não sou reconhecido; Se fizesse o serviço que realizo aqui num consul- \\
tório particular a remuneração seria muito melhor; A minha remuneração como dentista \\
no serviço público é inadequada e causa insatisfação; O reconhecimento do paciente com \\
relação ao atendimento não é o bastante para o dentista, os governantes precisam saber \\
que temos que estar satisfeitos com o nosso salário; Para sobreviver tenho que ter vários \\
trabalhos; Com o salário que ganho não tenho condições, não dá pra fazer cursos, ir a \\
congressos; As autoridades não estão preocupadas com isso.
\end{tabular}

Entretanto, estudos que abordam a remuneração entendem que o reembolso tradicional pela prestação de serviço deve ter papel não apenas de variável econômica, mas também de recompensa ou fator motivacional nos ambientes de trabalho, principalmente no que diz respeito à remuneração $0^{4}$.

Com os efeitos ocasionados pela inexistência, inadequação e/ou desatualização dos planos de carreira, especialmente no Sistema Único de Saúde (SUS), percebeu-se a necessidade das reivindicações pelas entidades de classes, visando à implementação dos planos de carreira na administração pública ${ }^{5}$.
O Quadro 2 apresenta a concepção dos cirurgiões-dentistas sobre os agravos e a falta de assistência à saúde como fator de interferência na qualidade de vida. No Brasil, estudos têm demonstrado e contextualizado a saúde do trabalhador brasileiro, enfatizando os índices elevados de acidentes de trabalho e doenças ocupacionais ${ }^{6}$.

A situação de trabalho comporta aspectos de ordem material, organizacional e humana que necessitam ser avaliados individualmente, em busca de melhores condições de adaptação entre o homem e o trabalho ${ }^{6}$.

Quadro 2 - IC 2 - Agravos e falta de assistência à saúde como fator de interferência da qualidade de vida dos cirurgiões-dentistas

\begin{tabular}{|c|l|}
\hline 2. IDEIA CENTRAL & \multicolumn{1}{c|}{ Discurso do Sujeito Coletivo } \\
\hline \multirow{5}{*}{ Sou a própria doença. } & $\begin{array}{l}\text { Sou a própria doença; Como dentista adquiri tendinite, escoliose, dores que me limitam } \\
\text { a determinados exerćcios; Dores cervicais, lombares e musculares diariamente; Adquiri a } \\
\text { Síndrome do Túnel do Carpo, fiz a cirurgia, mas como está inflamado ainda me incomoda; } \\
\text { Estou com perda auditiva por causa do barulho do motor de rotação, que é alto; Tive } \\
\text { AVC porque não sabia que era hipertenso; Tenho dificuldade para dormir, chego em casa } \\
\text { irritado e estressado por causa do trabalho; O trabalho devia [sic] ter políticas para prevenir } \\
\text { doenças; Ter acompanhamento mais de perto; Acompanhamento psicológico, ter umas } \\
\text { terapias de grupo para relaxar, porque a assistência pública vai de mal a pior; Esperar pra } \\
\text { ter assistência pelo SUS é complicado; Quando preciso de assistência tenho que procurar } \\
\text { por meio do plano de saúde; Se não tivesse plano de saúde iria ser péssimo, devido aos } \\
\text { profissionais da rede, que não são bem remunerados e dificultam o trabalho ao público. }\end{array}$ \\
\hline
\end{tabular}

O Quadro 3 apresenta a concepção dos cirurgiões-dentistas sobre a segurança e a higiene no trabalho. Estudiosos nessa temática atribuem a fatores como exposição contínua a condições físicas, mecânicas e psíquicas inadequadas no ambiente de trabalho um dos motivos para o aparecimento de distúrbios ao sistema musculoesquelético ${ }^{7}$.

Quadro 3 - IC 3 - Segurança e higiene no trabalho como fator de interferência da qualidade de vida dos cirurgiões-dentistas

\begin{tabular}{|l|l|}
\hline 3. IDEIA CENTRAL & \multicolumn{1}{|c|}{ Discurso do Sujeito Coletivo } \\
\hline Falta material para trabalhar. & $\begin{array}{l}\text { Os riscos poderiam ser evitados se os equipamentos daqui estivessem funcionando } \\
\text { adequadamente; As luvas, máscaras e gorro, apesar de serem fornecidos, sua qualidade } \\
\text { fica a desejar; Falta material para trabalhar, tem a questão da higiene... falta papel para } \\
\text { limpar as mãos, tem o ar-condicionado que precisa ser limpo; Solicitei protetor auricular } \\
\text { e nunca fui ouvida, por isso tive de comprar; Imagine trabalhar sem materiais! A pessoa } \\
\text { fica irritada, estressada; já me acostumei com o ruído e não estou mais nem notando a } \\
\text { sua existência; Detectei um vazamento no raio-X, comuniquei a coordenação, mas ele } \\
\text { só foi trocado de sala... Se reclamar muito, ficam dizendo que estamos inventando e } \\
\text { não queremos trabalhar; A relação interpesoal é pouca, pelo fato de estarmos trancados } \\
\text { entre quatro paredes, sem ter contato com os outros colegas; A carga horária deveria [ser] } \\
\text { reduzida e evitaria tensão; }\end{array}$ \\
\hline
\end{tabular}


Carga excessiva de trabalho, horas extras, ritmo acelerado, deficit de trabalhadores, equipamentos inadequados, iluminação insuficiente, sobrecargas referentes à repetitividade e postura inadequada são fatores de risco que estão intrinsecamente relacionados com os distúrbios musculoesqueléticos ${ }^{8}$.

A legislação brasileira determinou o modelo de transferência de recursos federais para os governos subnacionais, visando a financiar as ações e os serviços descentralizados de saúde ${ }^{9}$. Essa determinação deve ser regular e automática, observando critérios sociais, epidemiológicos, características da rede de serviços oferecidos, níveis de desempenho, entre outros, para que sejam destinados a investimentos na rede de serviço, na cobertura assistencial e em outras ações e serviços de saúde.
Desse modo, percebe-se, por meio dos discursos, a necessidade de investimentos no ambiente de trabalho, focando em melhorias das instalações, na compra e distribuição de equipamentos de proteção individual e materiais de consumo, em ações preventivas e na assistência à saúde, objetivando a diminuição dos riscos ocupacionais aos quais os cirurgiões-dentistas estão expostos.

O Quadro 4 apresenta a concepção dos cirurgioes-dentistas sobre a qualidade de vida (QV) como sinônimo de realização das múltiplas dimensões do viver. A QV das pessoas vem provocando reflexões teóricas e interferindo na vida das populações. Dessa forma, a avaliação da $\mathrm{QV}$ tornou-se fundamental para a compreensão do processo saúde-doença ${ }^{10}$.

Quadro 4 - IC 4 - A percepção da qualidade de vida como sinônimo de realização das múltiplas dimensões do viver

\begin{tabular}{|l|l|}
\hline \multicolumn{1}{|c|}{$4^{\text {a IDEIA CENTRAL }}$} & \multicolumn{1}{c}{ Discurso do Sujeito Coletivo } \\
\hline \multirow{5}{*}{ Viver bem. } & $\begin{array}{l}\text { É você ter saúde, ter lazer; Ter um bom emprego; Ter um bom salário para que possa se } \\
\text { manter; Poder desenvolver um trabalho satisfatório sem ter desgaste físico e mental; Fazer } \\
\text { o que gosta em casa e no trabalho; Ter paz; Ter uma boa família e uma boa moradia; } \\
\text { Estar bem com Deus e consigo mesmo; É ter boas relações sociais; É ser feliz; Hoje não } \\
\text { tenho qualidade de vida porque não estou estabilizado financeiramente, mas espero } \\
\text { conseguir muita coisa e ter qualidade de vida. }\end{array}$ \\
\hline
\end{tabular}

Em outra conceituação, a QV é descrita como uma variante entre indivíduos, classes sociais, regiões, situações, épocas e culturas, entretanto, ela atua de forma decisiva na vida dos indivíduos nos mais diversos ambientes sociais ${ }^{11}$.

Embora não haja um consenso a respeito de sua definição, a Organização Mundial de Saúde (OMS) conceitua a QV como a percepção do indivíduo de sua posição na vida, no contexto de sua cultura e no sistema de valores nos quais ele vive e em relação à suas expectativas, padrões e preocupações ${ }^{12}$.

Esse amplo conceito atribui à terminologia as suas principais características: subjetividade; multidimensionalidade, que contempla as dimensões físicas, psicológicas e social; e bipolaridade ${ }^{12}$. No ambiente de trabalho, esse termo é utilizado para descrever fatores humanos e ambientais, geralmente negligenciados pelas organizações em decorrência do avanço tecnológico, da produção e do crescimento econômico ${ }^{13}$.

Apesar dos conceitos atribuídos à $\mathrm{QV}$, é fundamental que o indivíduo seja visto de forma holística, pois se acredita que um baixo desempenho no ambiente de trabalho está intimamente associado a uma baixa qualidade de vida geral ${ }^{14}$.

Um bom ambiente de trabalho permite, entre outras coisas, que os trabalhadores tenham outros compromissos, tais como os relacionados à família, às relações sociais e aos hobbies ${ }^{15}$.

Pode-se verificar, por meio do Discurso do Sujeito Coletivo, uma similaridade entre este e o que é definido pela OMS. No entanto, nessas mesmas falas, também fica explícita a insatisfação dos profissionais pesquisados com o trabalho no que refere à sua instabilidade e remuneração salarial.

\section{Conclusões}

Esta pesquisa fez perceber os riscos, patologias e agravos à saúde dos cirurgiões-dentistas em sua rotina diária de trabalho. Revendo o objetivo proposto, pode-se observar que o cirurgião-dentista apresenta uma grande chance de ser acometido por agravos à saúde e doenças decorrentes de sua atividade, por estar exposto aos mais diversos riscos ocupacionais.

Verifica-se, por meio do Discurso do Sujeito Coletivo, que a fala dos cirurgiões-dentistas, antes individualizada, ganha corpo ao generalizar as suas interferências na qualidade de vida em decorrência da exposição diária e contínua aos riscos e às patologias ocupacionais. Percebe-se, assim, uma insatisfação generalizada por parte dos trabalhadores com relação à carga excessiva de trabalho e remuneração, a qual além de ser incompatível com os serviços oferecidos pelas clínicas privadas, diverge entre as esferas de governo. Tal insatisfação poderá ser minimizada com a aprovação das Diretrizes Nacionais para a Instituição de Planos de Carreiras, Cargos e Salários no âmbito do Sistema Único de Saúde. 


\section{Abstract}

Objective: to identify occupational risks, injuries, diseases, and pathologies to which dental surgeons are exposed, and verify the interference of these factors in the quality of life of participants of the study. Method: descriptive study with quantitative and qualitative approach, held at the Center for Dental Specialties (CDS) located in the city of João Pessoa, PB, Brazil. Results: the results revealed by the Collective Subject Discourse showed great interference caused by occupational risk, especially the dissatisfaction with pay, safety, and hygiene in the workplace. Conclusion: despite claiming to have quality of life, most professionals presented feelings of dissatisfaction with health and work.

Keywords: Occupational risks. Occupational pathologies. Quality of life.

\section{Referências}

1. Amarante ST. Análise das condições ergonômicas do trabalho de enfermeiras de centro cirúrgico [Dissertação de Mestrado]. São Paulo: Escola de Enfermagem da Universidade de São Paulo; 1999.

2. Moscovici SA. Representação social da psicanálise. Rio de Janeiro: Zahar; 1978.

3. Hernandez JB, Coulter I, Goldman D, Freed J, Marcus M. Managed care in dental markets: is the experience of medicine relevant? J Public Health Dent 1999; 59:24-32.

4. Batista AAV, Vieira MJ, Cardoso NCS, Carvalho GRP. Fatores de motivação e insatisfação no trabalho do enfermeiro. Rev Esc Enferm USP 2005; 39(1):85-91.

5. Brasil. PCCS - SUS: Diretrizes Nacionais para a Instituição de Planos de Carreiras, Cargos e Salários no Âmbito do Sistema Único de Saúde. Brasília: Ministério da Saúde; 2006.

6. Marziale MHP. Condições ergonômicas da situação de trabalho, do pessoal de enfermagem, em uma unidade de internação hospitalar [Tese de Doutorado]. Ribeirão Preto: Escola de Enfermagem de Ribeirão Preto da Universidade de São Paulo; 1995.

7. Parada EO, Alexandre NMC, Benatti MCC. Lesões ocupacionais afetando a coluna vertebral em trabalhadores de enfermagem. Rev Latino-Am Enfermagem [periódico online] 2002 Jan [citado 2013 Oct 24]; 10(1):64-9. Disponível em URL: http://www.scielo.br/scielo.php?script=sci_ arttext\&pid=S0104-11692002000100010\&lng=en.

8. Mauro CCC, Mauro MYC, Cupello AJ. O trabalho de enfermagem hospitalar: uma visão ergonômica [CD-ROM]. Gramado: ABERGO; 2001.

9. Lima LD. Conexões entre o federalismo fiscal e o financiamento da política de saúde no Brasil. Ciênc Saúde Colet [periódico online]. 2007 Apr [citado 2013 Oct 24]; 12(2): 511-522. Disponível em URL: http://www. scielo.br/scielo.php?script=sci_arttext\&pid=S1413$-81232007000200027 \& \operatorname{lng}=\mathrm{en}$.

10. Costa SPR. Perfil da qualidade de vida de portadores de colostomia [Dissertação de Mestrado]. João Pessoa: Centro de Ciências e Sáude da Universidade Federal de Sergipe; 2007.
11. Fleck MPA. A avaliação de qualidade de vida: guia para profissionais de saúde. Porto Alegre: Artmed; 2008.

12. OMS. Whoqol Group. The World Health Organization Quality of Life Assessment: position paper from the world health organization. Genebra: OMS; 1995.

13. Walton RE. Quality of work life: what is it? Sloan Management Review 1973; 1:11-21.

14. Seidl EMF, Zannon CMLC. Qualidade de vida e saúde: aspectos conceituais e metodológicos. Cad Saúde Pública [periódico online]. 2004 Apr [citado 2013 Oct 24]; 20(2):580-588. Disponível em URL: http://www.scielo.br/scielo.php?script=sci_ arttext\&pid=S0102-311X2004000200027\&lng=en. http://dx. doi.org/10.1590/S0102-311X2004000200027.

15. Levering $\mathrm{R}$. Um excelente lugar para se trabalhar: o que torna alguns empregadores tão bons (e outros tão ruins). Rio de Janeiro: Qualitymark; 1986.

\section{Endereço para correspondência:}

Rua José Francisco da Silva, 651, Cristo Redentor

58070-410 João Pessoa / PB

Fone: 7991429083

E-mail: marlos.noronha@gmail.com

Recebido: 24/10/2013. Aceito: 16/01/2014. 